\title{
A Fast Method for Pricing Early-Exercise Options with the FFT
}

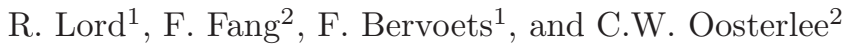 \\ ${ }^{1}$ Modeling and Research, Rabobank International, Utrecht, The Netherlands \\ roger.lord@rabobank.com; frank.bervoets@rabobank.com \\ ${ }^{2}$ Delft University of Technology, Delft Institute of Applied Mathematics, Delft, \\ The Netherlands \\ f.fang@ewi.tudelft.nl; c.w.oosterlee@tudelft.nl
}

\begin{abstract}
A fast and accurate method for pricing early exercise options in computational finance is presented in this paper. The main idea is to reformulate the well-known risk-neutral valuation formula by recognizing that it is a convolution. This novel pricing method, which we name the 'CONV' method for short, is applicable to a wide variety of payoffs and only requires the knowledge of the characteristic function of the model. As such the method is applicable within exponentially Lévy models, including the exponentially affine jump-diffusion models. For an $M$-times exercisable Bermudan option, the overall complexity is $O(M N \log (N))$ with $N$ grid points used to discretize the price of the underlying asset. It is also shown that American options can be very efficiently computed by combining Richardson extrapolation to the CONV method.
\end{abstract}

Keywords: Option pricing, Lévy Process, Convolution, FFT, Transform.

\section{Introduction}

When valuing and risk-managing exotic derivatives, practitioners demand fast and accurate prices and sensitivities. As the financial models and option contracts used in practice are becoming increasingly complex, efficient methods have to be developed to cope with such models. Aside from non-standard exotic derivatives, plain vanilla options in many stock markets are actually of the American type. As any pricing and risk management system has to be able to calibrate to these plain vanilla options, it is of the utmost importance to be able to value these American options quickly and accurately.

In the past couple of years a vast body of literature has considered the modeling of asset returns as infinite activity Lévy processes, due to the ability of such processes to adequately describe the empirical features of asset returns and at the same time provide a reasonable fit to the implied volatility surfaces observed in option markets. Valuing American options in such models is however far from trivial, due to the weakly singular kernels of the integral terms appearing in the PIDE, as reported in, e.g., 261011. 
In this paper we present a novel FFT-based method for pricing options with early exercise features. The only requirement of the method is that the characteristic function of the underlying asset is known, which is the case for many exponential Lévy model, with the popular exponentially affine jump-diffusion (EAJD) models of [7] as an important subclass. In contrast to the PIDE methods, our method has no difficulty in handling processes of infinite activity, such as the Variance Gamma (VG) or CGMY models. A real benefit of this class of methods is, next to its flexibility, the impressive computational speed, as the FFT algorithm is employed.

\section{FFT-Based Methods for Option Pricing in Literature}

All transform methods depart from the risk-neutral valuation formula that, for a European option, reads:

$$
V(t, S(t))=e^{-r \tau} \mathbf{E}[V(T, S(T))]
$$

where $\mathbf{E}$ denotes the operator of taking expectation of some random variable w.r.t. risk-neutral probability measure, $V$ denotes the value of the option, $r$ is the risk-neutral interest rate $1, t$ is the current time point, $T$ is the maturity of the option and $\tau=T-t$. The variable $S$ denotes the underlying asset price.

Since for many models the density is not available in closed-form whereas the characteristic function is, a number of papers starting from Heston [9] have attacked the problem via another route. Focusing on a plain vanilla European call option, note that for dividend-protected assets (1) can be written very generally as:

$$
V(t, S(t))=S(t) \cdot \Delta-K e^{-r(T-t)} \mathbb{P}(S(T)>K),
$$

where $\mathbb{P}(S(T)>K)$ is the risk-neutral probability of ending up in-the-money and $\Delta$ is the delta of the option, the sensitivity of the option with respect to changes in the stock price. Both $\mathbb{P}(S(T)>K)$ and $\Delta$ can be recovered by inverting techniques, e.g., by Gil-Palaez inversion [8]. Carr and Madan [4] considered another approach by directly taking the Fourier transform of the damped option price with respect to $k$, the logarithm of the strike price. Premultiplying the option price with a damping function $\exp (\alpha k)$ to ensure the existence of the Fourier transform, Carr and Madan ended up with

$$
\mathcal{F}\left\{e^{\alpha k} V(t, k)\right\}=e^{-r \tau} \int_{\mathbb{R}} e^{i u k} \mathbf{E}\left[\left(S(T)-e^{k}\right)^{+}\right] d k=\frac{e^{-r \tau} \phi(u-(\alpha+1) i)}{-(u-\alpha i)(u-(\alpha+1) i)},(3)
$$

where $i$ the imaginary unit, $k$ is the logarithm of the strike price $K$ and $\phi$ is the characteristic function of the log-underlying, i.e., $\phi(u)=\mathbf{E}\left[e^{i u \ln S(T)}\right]$. The methods considered up till here can only handle the pricing of European options.

${ }^{1}$ Throughout the paper we assume that interest rates are deterministic, this assumption can be relaxed at the cost of increasing the dimensionality of some of the methods. 
Define the set of exercise dates as $\mathcal{T}=\left\{t_{0}, \ldots, t_{M}\right\}$ and assume the exercise dates are equally spaced: $t_{k+1}-t_{k}=\Delta t$. The best known examples of early exercise options are the American and Bermudan options. American options can be exercised at any time prior to the option's expiry; Bermudan options can only be exercised at certain dates in the future. The Bermudan option price can then be found via backward induction as

$$
\left\{\begin{array}{l}
C\left(t_{k}, S\left(t_{k}\right)\right)=e^{-r \Delta t} \mathbf{E}\left[V\left(t_{k+1}, S\left(t_{k+1}\right)\right)\right] \\
V\left(t_{k}, S\left(t_{k}\right)\right)=\max \left\{C\left(t_{k}, S\left(t_{k}\right)\right), E\left(t_{k}, S\left(t_{k}\right)\right)\right\},
\end{array} \quad k=M-1, \ldots, 0,\right.
$$

where $C$ denotes the continuation value of the option and $V$ is the option value on the very next exercise date. Clearly the dynamic programming problem in (4) is a successive application of the risk-neutral valuation formula, as we can write the continuation value as

$$
C\left(t_{k}, S\left(t_{k}\right)\right)=e^{-r \Delta t} \int_{\mathbb{R}} V\left(t_{k+1}, y\right) f\left(y \mid S\left(t_{k}\right)\right) d y,
$$

where $f\left(y \mid S\left(t_{k}\right)\right)$ represents the probability density describing the transition from $S\left(t_{k}\right)$ at $t_{k}$ to $y$ at $t_{k+1}$. Based on (4) and (5) the QUAD method was introduced in [1]. The method requires that the transition density is known in closed-form. This requirement is relaxed in [12], where the QUAD-FFT method is introduced and the underlying idea is that the transition density can be recovered by inverting the characteristic function. But the overall complexity of both methods is $O\left(M N^{2}\right)$ for an $M$-times exercisable Bermudan option with $N$ grid points used to discretize the price of the underlying asset.

\section{The CONV Method}

One of the refining properties of a Lévy process is that its increments are independent of each other, which is the main premise of the CONV method:

$$
f(y \mid x)=f(y-x) .
$$

Note that $x$ and $y$ do not have to represent the asset price directly, they could be monotone functions of the asset price. The assumption made in (6) therefore certainly holds when the asset price is modeled as a monotone function of a Lévy process, since one of the defining properties of a Lévy process is that its increments are independent of each other. In this case $x$ and $y$ in (6) represent the log-spot price. By including (6) in (5) and changing variables $z=y-x$ the continuation value can be expressed as

$$
C\left(t_{k}, x\right)=e^{-r \Delta t} \int_{-\infty}^{\infty} V\left(t_{k+1}, x+z\right) f(z) d z
$$

which is a cross-correlation of the option value at time $t_{k+1}$ and the density $f(z)$. If the density function has an easy closed-form expression, it may be beneficial 
to compute the integral straight forwardly. However, for many exponential Lévy models we either do not have a closed-form expression for the density (e.g. the CGMY/KoBoL model of [3] and many EAJD models), or if we have, it involves one or more special functions (e.g. the Variance Gamma model).

Since the density is hard to obtain, let us consider taking the Fourier transform of (7). In the remainder we will employ the following definitions for the continuous Fourier transform and its inverse,

$$
\begin{aligned}
\hat{h}(u) & :=\mathcal{F}\{h(t)\}(u)=\int_{-\infty}^{\infty} e^{-i u t} h(t) d t, \\
h(t) & :=\mathcal{F}^{-1}\{\hat{h}(u)\}(t)=\frac{1}{2 \pi} \int_{-\infty}^{\infty} e^{i u t} \hat{h}(u) d u .
\end{aligned}
$$

If we dampen the continuation value (7) by a factor $\exp (\alpha x)$ and subsequently take its Fourier transform, we arrive at

$$
e^{r \Delta t} \mathcal{F}\left\{e^{\alpha x} C\left(t_{k}, x\right)\right\}(u)=\int_{-\infty}^{\infty} e^{-i u x} e^{\alpha x} \int_{-\infty}^{\infty} V\left(t_{k+1}, x+z\right) f(z) d z d x .
$$

Changing the order of the integrals and the variables by $x=y-z$, we obtain

$$
\begin{aligned}
e^{r \Delta t} \mathcal{F}\left\{e^{\alpha x} C\left(t_{k}, x\right)\right\}(u) & =\int_{-\infty}^{\infty} \int_{-\infty}^{\infty} e^{-i(u+i \alpha) y} V\left(t_{k+1}, y\right) d y e^{i(u+i \alpha) z} f(z) d z \\
& =\int_{-\infty}^{\infty} e^{-i(u+i \alpha) y} V\left(t_{k+1}, y\right) d y \int_{-\infty}^{\infty} e^{i(u+i \alpha) z} f(z) d z \\
& =\mathcal{F}\left\{e^{\alpha y} V\left(t_{k+1}, y\right)\right\}(u) \phi(u+i \alpha) .
\end{aligned}
$$

In the last step we used the fact that the complex-valued Fourier transform of the density is simply the extended characteristic function

$$
\phi(x+y i)=\int_{-\infty}^{\infty} e^{i(x+y i) z} f(z) d z
$$

which is well-defined when $\phi(-y i)<\infty$, as $|\phi(x+y i)| \leq|\phi(-y i)|$. Inverse Fourier transform and undamping on (11) yield the CONV formula:

$$
C\left(t_{k}, x\right)=e^{-r \Delta t-\alpha x} \mathcal{F}^{-1}\left\{\mathcal{F}\left\{e^{\alpha y} V\left(t_{k+1}, y\right)\right\}(u) \cdot \phi(u+i \alpha)\right\} .
$$

To value Bermudan options, one can recursively call (13) and (4) backwards in time: First recover the option values on the last early-exercise date; then feed them into (13) and (4) to obtain the option values on the second last early-exercise date; $\cdots$, continue the procedure till the first early-exercise date is reached; for the last step, feed the option value on the first early-exercise date into (13) and there we obtain the option values on the initial date.

To value American options, there are two routes to follow: they can be approximated either by Bermudan options with many early exercise dates or by Richardson extrapolation based on only a series of Bermudan options with an 
increasing number of early exercise dates. In the experiments we evaluated both approaches and compared their CPU time and the accuracy. As for the approach via Richardson extrapolation, our choice of scheme is the one proposed by Chang, Chung, and Stapleton [5].

\section{Implementation}

Let's ignore the damping factor in this section, for the ease of analysis, and simplify the notations as: $e^{-r \Delta t} C\left(x, t_{k}\right) \rightarrow C(x)$ and $V\left(y, t_{k+1}\right) \rightarrow V(y)$.

Suppose that we are only interested in a portion of $C(x)$ that lies in $\mathcal{T}_{L}:=$ $\left[-\frac{L}{2}, \frac{L}{2}\right]$. Assume that $f(z) \approx 0$ for $z$ outside $\mathcal{T}_{A}:=\left[-\frac{A}{2}, \frac{A}{2}\right]$. Both $L$ and $A$ denote positive real numbers. Then we may re-write the risk-neutral valuation formula as

$$
C(x)=\int_{\mathbb{R}} V(x+z) f(z) d z=\int_{\mathcal{T}_{A}} V(x+z) f(z) d z,
$$

which indicates that if values of $C(x)$ are wanted on $\mathcal{T}_{L}$ then values of $V(y)$ that we need for computation lie in $\mathcal{T}_{A+L}:=\left[-\frac{L+A}{2}, \frac{L+A}{2}\right]$.

Remark 1 (Value of $A$ ). When working in the log-stock domain (e.g. $x:=$ $\log (S)$ ), we approximate the standard deviation of the density function by the volatility of its characteristic function, therefore approximate $A$ by 10 times volatility. The approximation gives good results in series of experiments.

\subsection{Discrete CONV Formula}

Recall that functions on compact supports can be represented by their Fourier series, it then follows that we may rewrite $V(y)$ as

$$
V(y)=\sum_{k \in Z Z} v_{k} e^{i k \frac{2 \pi}{A+L} y} \text {, with } v_{k}=\frac{1}{A+L} \int_{\mathcal{T}_{A+L}} V(y) e^{-i k \frac{2 \pi}{A+L} y} d y
$$

Substitute the Fourier series of $V(y)$ in (14) and interchange the summation and the integration (allowed by Fubini's theorem) to result in

$$
C(x)=\sum_{k \in Z} v_{k}\left[\int_{\mathcal{T}_{A}} f(z) e^{i k \frac{2 \pi}{A+L} z} d z\right] e^{i k \frac{2 \pi}{A+L} x}
$$

where the integration inside the brackets is precisely the definition of the characteristic function at $u=k \frac{2 \pi}{A+L}$. Truncate the series in (16) to yield

$$
\widetilde{C}(x)=\sum_{k \in \mathcal{Z}_{N}} v_{k} \cdot \phi\left(k \frac{2 \pi}{A+L}\right) \cdot e^{i k \frac{2 \pi}{A+L} x},
$$


where $\mathcal{Z}_{N}=\left\{n \mid-\frac{N}{2} \leq n<\frac{N}{2}, \in \mathbb{Z}\right\}$. Up to this point, (17) is almost ready for the implementation, were $v_{k}$ to be obtained numerically as well. To recover $v_{k}$, quadrature rules are employed. With composite mid-point rule one obtains

$$
\tilde{v}_{k}=\frac{\Delta y}{L+A} \sum_{j \in \mathcal{Z}_{N}} e^{-i k \frac{2 \pi}{L+A} y_{j}} V\left(y_{j}\right)
$$

where $\Delta y=\frac{L+A}{N},\left\{y_{j}:=j \Delta y+y_{c} \mid j \in \mathcal{Z}_{N}\right\}$ and $y_{c}$ denotes the grid center. It then yields the discrete version of the CONV formula after substituting (18) into (17):

$$
C_{m}=\frac{1}{N} \sum_{k \in \mathcal{Z}_{N}} e^{i u_{k} x_{m}} \phi\left(u_{k}\right) \sum_{j \in \mathcal{Z}_{N}} e^{-i u_{k} y_{j}} V\left(y_{j}\right),
$$

where $u_{k}=k \frac{2 \pi}{L+A}$ and $\left\{x_{m}:=m \Delta y+x_{c} \mid m \in \mathcal{Z}_{N}\right\}$ with grid center $x_{c}$. Note that the $x$ - and $y$-grids share the same mesh size so that the same $u$-grid can be used in both the inner and the outer summations.

\subsection{Computational Complexity and Convergence Rate}

The pleasing feature of (19) is that both summations can be fast resolved by existing FFT algorithms. Therefore, the overall computational complexity is $\mathcal{O}(N \log (N))$ for European options, and $\mathcal{O}(M N \log (N))$ for an $M$-times exercisable Bermudan options.

In the mean while, it can be proven analytically that the convergence rate of the method is $\mathcal{O}\left(\frac{1}{N^{2}}\right)$ for both vanilla European and Bermudan options. Though we'll not include the error analysis in this paper, the regular point-wise convergence rate of the method can be well observed in the experiment results.

\section{$5 \quad$ Numerical Results}

By various numerical experiments we aim to show the speed of the computations and the flexibility of the CONV method. Three underlying models are adopted in the experiments: Geometric Brownian Motion (GBM), Variance Gamma (VG), and CGMY. The pricing problems are of Bermudan and American style.

The computer used for the experiments has a Intel Pentium $4 \mathrm{CPU}, 2.8 \mathrm{GHz}$ frequency and a total $1011 \mathrm{MB}$ physical memory. The code is programmed in Matlab.

Results for 10-times exercisable Bermudan options under GBM and VG are summarized in table 1, where the fast computational speed (e.g. less than 1 second for $N=2^{16}$ ), the high accuracy (e.g. with only $2^{6}$ grid points the error is already of level $10^{-2}$ ) and the regular convergence rate (e.g. the convergence rate is 4 for Bermudan payoff) are shown. Results for American options under VG and CGMY are summarized in table 2, where ' $\mathrm{P}(\mathrm{N} / 2)$ ' denotes the results obtained by approximating the American option values directly by $N / 2$-times exercisable 
Table 1. CPU time, errors and convergence rate in pricing 10-times exercisable Bermudan put under GBM and VG with the CONV method

\begin{tabular}{c|c|c|c||c|c|c}
\hline \multirow{2}{*}{$N=2^{d}$} & \multicolumn{2}{|c||}{ GBM: Reference $=10.4795201 ;$} & \multicolumn{3}{c}{ VG: Reference $=9.04064611 ;$} \\
\cline { 2 - 7 } & time(sec) & absolute error & convergence & time(sec) & absolute error & convergence \\
\hline 6 & 0.002 & $9.54 \mathrm{e}-02$ & & 0.001 & $7.41 \mathrm{e}-02$ & \\
8 & 0.002 & $2.44 \mathrm{e}-02$ & 3.91 & 0.002 & $5.42 \mathrm{e}-03$ & 1.37 \\
9 & 0.003 & $6.45 \mathrm{e}-03$ & 3.78 & 0.003 & $2.68 \mathrm{e}-03$ & 2.02 \\
10 & 0.010 & $1.69 \mathrm{e}-03$ & 3.81 & 0.006 & $6.10 \mathrm{e}-04$ & 4.39 \\
11 & 0.011 & $4.47 \mathrm{e}-04$ & 3.79 & 0.015 & $1.38 \mathrm{e}-04$ & 4.40 \\
12 & 0.021 & $1.12 \mathrm{e}-04$ & 3.97 & 0.022 & $3.16 \mathrm{e}-05$ & 4.38 \\
13 & 0.043 & $2.83 \mathrm{e}-05$ & 3.97 & 0.042 & $7.92 \mathrm{e}-06$ & 3.99 \\
14 & 0.091 & $7.09 \mathrm{e}-06$ & 4.00 & 0.096 & $1.99 \mathrm{e}-06$ & 3.97 \\
\hline
\end{tabular}

For GBM: $S_{0}=100, K=110, T=1, \sigma=0.2, r=0.1, q=0$;

For VG: $S_{0}=100, K=110, T=1, \sigma=0.12, \theta=-0.14, \nu=0.2, r=0.1, q=0$;

Reference values are obtained by the PIDE method with 4 million grid points.

Table 2. CPU time, errors and convergence rate in pricing 10-times exercisable Bermudan put under VG and CGMY with the CONV method

\begin{tabular}{|c|c|c|c|c|c|c|c|c|}
\hline \multirow[b]{3}{*}{$N=2^{d}$} & \multicolumn{4}{|c|}{ VG: Reference $=0.800873607$} & \multirow{2}{*}{\multicolumn{2}{|c|}{$\begin{array}{c}\text { CGMY }(Y<1) \\
\text { Reference= } \\
0.112171[2] \\
\text { Richardson }\end{array}$}} & \multirow{2}{*}{\multicolumn{2}{|c|}{\begin{tabular}{|c|} 
CGMY $(Y>1)$ \\
Reference= \\
$9.2185249[13$ \\
Richardson \\
\end{tabular}}} \\
\hline & \multicolumn{2}{|c|}{$\mathrm{P}(\mathrm{N} / 2)$} & \multicolumn{2}{|c|}{\begin{tabular}{|l|} 
Richardson \\
\end{tabular}} & & & & \\
\hline & time(sec) & error & time $(\mathrm{sec})$ & error & time(sec) & error & time(sec) & error \\
\hline 7 & 0.01 & $4.61 \mathrm{e}-02$ & 0.03 & $4.51 \mathrm{e}-02$ & \begin{tabular}{|l|}
0.02 \\
\end{tabular} & $1.37 \mathrm{e}-02$ & 0.02 & $5.68 \mathrm{e}-01$ \\
\hline 8 & 0.04 & $6.47 \mathrm{e}-03$ & 0.05 & $1.36 \mathrm{e}-02$ & 0.04 & $2.08 \mathrm{e}-03$ & 0.04 & $2.78 \mathrm{e}-01$ \\
\hline 9 & 0.11 & $6.78 \mathrm{e}-03$ & 0.07 & $2.69 \mathrm{e}-03$ & 0.07 & $4.83 \mathrm{e}-04$ & 0.08 & $1.29 \mathrm{e}-01$ \\
\hline 10 & 0.45 & $5.86 \mathrm{e}-03$ & 0.14 & $1.43 \mathrm{e}-03$ & 0.12 & $9.02 \mathrm{e}-05$ & 0.14 & $8.68 \mathrm{e}-03$ \\
\hline 11 & 1.73 & $2.87 \mathrm{e}-03$ & 0.28 & $2.71 \mathrm{e}-04$ & 0.26 & $4.21 \mathrm{e}-05$ & 0.28 & $6.18 \mathrm{e}-04$ \\
\hline 12 & 7.18 & $1.03 \mathrm{e}-03$ & 0.57 & $5.76 \mathrm{e}-05$ & 0.55 & $2.20 \mathrm{e}-05$ & 0.59 & $6.14 \mathrm{e}-03$ \\
\hline
\end{tabular}

For VG: $S_{0}=100, K=90, T=1, \sigma=0.12, \theta=-0.14, \nu=0.2, r=0.1, q=0$;

Reference value from PIDE implementation with about $65 \mathrm{~K} \times 16 \mathrm{~K}$ grid points

For $\operatorname{CGMY}(Y<1): Y=0.5, C=1, G=M=5, S_{0}=1, K=1, T=1, r=0.1, q=0$;

For $\operatorname{CGMY}(Y>1): Y=1.0102, C=0.42, G=4.37, M=191.2, S_{0}=90, K=98, T=$ $0.25, r=0.06, q=0$;

Bermudan options, and 'Richardson' denotes the results obtained by the 6 -times repeated Richardson extrapolation scheme. For the VG model, the extrapolation method turns out to converge much faster and spend far less time than the direct approximation approach (e.g., to get the same $10^{-4}$ accuracy, the extrapolation method is more than 20 times faster than the direct-approximation method). For CGMY model, results by the extrapolation approach are given. They demonstrate that the CONV method can be well combined with the extrapolation technique as well as any models with known characteristic functions. 


\section{Conclusions and Future Works}

The CONV method, like other FFT-based methods, is quite flexible w.r.t the choice of asset process and also the type of option contract. It can be applied if the underlying follows a Lévy processe and its characteristic function is known. The CONV method is highly accurate and fast in pricing Bermudan and American options. It can be used for fast option pricing and for parameter calibration purposes.

The future works include thorough error analysis and application of the method to exotic options. Generalization of the method to high-dimensions and incorporation of the method with sparse grid method are also of our great interest.

\section{References}

1. Andricopoulos, A.D., Widdicks, M., Duck, P.W. And Newton, D.P.: Universal Option Valuation Using Quadrature, J. Financial Economics, 67(2003),3: 447-471

2. Almendral, A. And Oosterlee, C.W.: Accurate Evaluation of European and American Options Under the CGMY Process., to appear in SIAM J. Sci. Com$\operatorname{put}(2006)$

3. Boyarchenko, S. I. And Levendorskĭ, S. Z.: Non-Gaussian Merton-BlackScholes theory, vol. 9 of Advanced Series on Statistical Science \& Appl. Probability, World Scientific Publishing Co. Inc., River Edge, NJ, 2002

4. CARR, P. P. And Madan, D. B.: Option valuation using the Fast Fourier Transform, J. Comp. Finance, 2 (1999), pp. 61-73

5. Chang, C-C, Chung, S-L and Stapleton, R.C.: Richardson extrapolation technique for pricing American-style options Proc. of 2001 Taiwanese Financial Association, Tamkang University Taipei, June 2001. Available at http://papers.ssrn. com/sol3/papers.cfm?abstract_id=313962

6. Cont, R. And Tankov, P.: Financial modelling with jump processes, Chapman \& Hall, Boca Raton, FL, 2004

7. Duffie, D., Pan, J. And Singleton, K.: Transform analysis and asset pricing for affine jump-diffusions. Econometrica 68(2000): 1343-1376

8. Gil-Pelaez, J.: Note on the inverse theorem. Biometrika 37(1951): 481-482

9. Heston, S.: A closed-form solution for options with stochastic volatility with applications to bond and currency options, Rev. Financ. Stud., 6 (1993), pp. 327-343.

10. Hirsa, A. And Madan, D. B.: Pricing American Options Under Variance Gamma, J. Comp. Finance, 7 (2004).

11. Matache, A. M., Nitsche, P. A. And Schwab, C.: Wavelet Galerkin pricing of American options on Lévy driven assets, working paper, ETH, Zürich, 2003.

12. O'Sullivan, C.: Path Dependent Option Pricing under Levy Processes EFA 2005 Moscow Meetings Paper, Available at SSRN: http://ssrn.com/abstract $=673424$, Febr. 2005.

13. WAng, I., Wan, J.W. And Forsyth, P. : Robust numerical valuation of European and American options under the CGMY process. Techn. Report U. Waterloo, Canada, 2006. 